\title{
Kontinuierlich besser
}

Entscheidende technische Fortschritte finden aktuell auf mehreren Gebieten gleichzeitig statt, die die Industriestrukturen, wie wir sie heute kennen, schon sehr bald entscheidend verändern werden. Neben Themen wie dem Internet der Dinge und künstlicher Intelligenz zählen viele Experten auch die generative Fertigung, auch Additive Manufacturing oder additive Fertigung genannt, dazu.

Die Technik ist nicht neu, erste Aktivitäten reichen bis in die 1950erJahre zurück. Es dauerte jedoch bis in die frühen 1980er-Jahre, bis die notwendigen Techniken wie etwa Computer und Laser zur Verfügung standen, um eine praktische Umsetzung möglich zu machen. Im Jahr 1984 wurden erste Patente für diese Art von Verfahren zeitgleich in Japan, Frankreich und den USA registriert. Seither wird die Technik weltweit beständig weiterentwickelt.

Ein großer Vorteil generativer Fertigungsverfahren zeigt sich zurzeit insbesondere bei Produkten, die auf konventionelle Art und Weise nur mühsam oder gar nicht hergestellt werden können. Der Leichtbau von Komponenten für Automobile und Flugzeuge ist ein typischer Anwendungsfall. So fertigt die BMW Group beispielsweise Steckdosen sowie Halterungen für Lichtleiter und Warnblinker für bestimmte Rolls-RoyceModelle generativ.
Die generative Fertigung wird mitunter als eine disruptive Technik bezeichnet, also als eine Innovation, die bestehende Techniken möglicherweise vollständig verdrängt. Damit entfesselt sie einerseits große Euphorie, ruft aber andererseits auch Argwohn in den Industriezweigen mit tradierten, fest etablierten Produktionsmethoden hervor. Das spiegelt sich auch in der Berichterstattung wieder. Die Technik polarisiert.

Im Hinblick auf die durchaus aktuell noch bestehenden Probleme der Verfahren - insbesondere die Wirtschaftlichkeit bei hohen Stückzahlen - ist die Branche allerdings emsig tätig. An vielen einzelnen Stellen werden bereits große Fortschritte im Detail gemacht, die helfen, generative Fertigungsverfahren und deren Prozesse zu verbessern. Insbesondere durch eine Zunahme an Kooperationen zwischen den Unternehmen und Forschungseinrichtungen, die sich damit beschäftigen, werden die Verfahren, die Softwaretools und passenden Werkstoffe in Zukunft kontinuierlich besser werden.

In dieser Sonderausgabe der lightweight.design möchten wir Ihnen einige Beispiele aus Industrie und Forschung vorstellen, die als Teil einer laufenden dynamischen Optimierung der generativen Verfahren einzuordnen sind. Wir wünschen Ihnen eine interessante Lektüre!

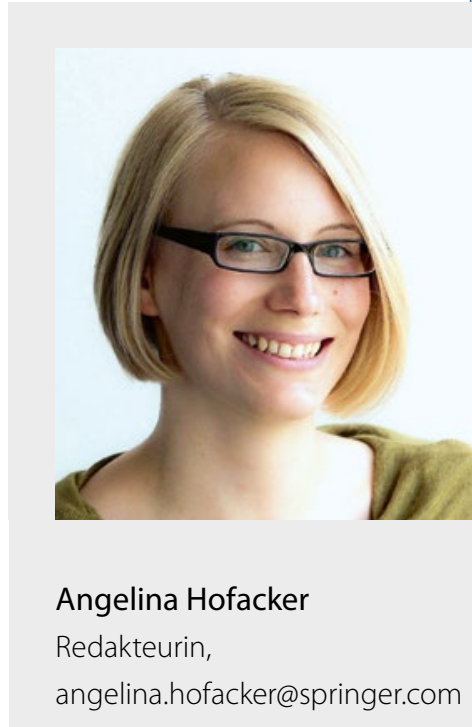

